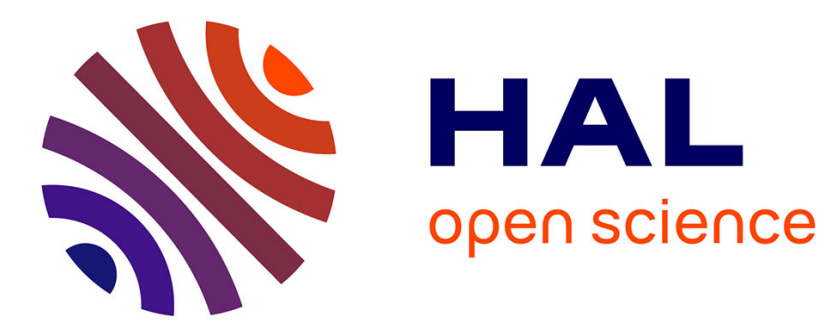

\title{
Écriture et prophétie en Israël à la fin de l'époque monarchique
}

\author{
Christophe Batsch
}

\section{To cite this version:}

Christophe Batsch. Écriture et prophétie en Israël à la fin de l'époque monarchique. Semitica et Classica, 2009, 2, pp.35-41. 10.1484/J.SEC.1.100506 . hal-01669285

\section{HAL Id: hal-01669285 \\ https://hal.univ-lille.fr/hal-01669285}

Submitted on 21 Dec 2017

HAL is a multi-disciplinary open access archive for the deposit and dissemination of scientific research documents, whether they are published or not. The documents may come from teaching and research institutions in France or abroad, or from public or private research centers.
L'archive ouverte pluridisciplinaire HAL, est destinée au dépôt et à la diffusion de documents scientifiques de niveau recherche, publiés ou non, émanant des établissements d'enseignement et de recherche français ou étrangers, des laboratoires publics ou privés. 


\section{CHRISTOPHE BATSCH Écriture et prophétie en Israël à la fin de l'époque monarchique}

Les prophètes contemporains de l'essor de l'écrit dans le royaume de Juda dont les textes sont regroupés sous les noms du Proto-Isaïe et de Jérémie, ont été confrontés à l'émergence d'une nouvelle technique de transmission des savoirs, d'exercice du pouvoir et d'affirmation de l'autorité ; cette technique dont ils n'ont pas la maîtrise s'installe très vite en position de concurrence efficace contre leurs propres outils de transmission et d'autorité, fondés sur l'oralité et le spectaculaire métaphorique. Plusieurs passages tirés de ces deux recueils prophétiques attestent le conflit qui se développe alors entre ces deux techniques d'expression, d'interprétation, de compréhension et de transmission de la volonté divine, fondement ultime de tous les pouvoirs religieux et politiques.

Ces passages, rares mais immensément instructifs sont :

1/ Le « miroir d'Isaïe » (Is 8, 1-4)

Où l'on voit le prophète Isaïe tenter de s'approprier et de détourner les techniques de l'écrit pour en faire un usage métaphorique et symbolique qui s'accorde à la tradition prophétique mais ignore les qualités propres, la portée et l'usage de l'écrit - toutes choses déjà acquises par la pratique des siècles précédents dans le royaume voisin d'Israël.

Une tentative analogue peut être repérée dans l'usage magico-symbolique d'un rouleau envoyé aux exilés de Babylone par Jérémie et noyé dans l'Euphrate après lecture (Jr 51, 59-64).

2/ Le « rouleau brûlé de Jérémie » (Jr 36 passim)

Où le conflit entre l'autorité de la loi écrite du roi et celle du discours prophétique est symboliquement tranché en faveur de la première par le roi Yoyakim, dans le geste de brûler le rouleau sur lequel ont été transcrites les prophéties de Jérémie au fur et à mesure qu'on lui en faisait la lecture; et où l'on peut observer que la mise par écrit des prophéties ne diminue ni ne modifie leur caractère essentiellement oral.

\section{LE « MIROIR D’ISAÏE »}

On sait que les prophètes d'Israël recourraient volontiers, outre à l'oralité des vaticinations prophétiques, à des modes d'expression symboliques mettant en scène leur propre corps : le prophète anonyme de Béthel tué par un lion $^{1}$; le prophète contre Achab mutilé et déguisé ${ }^{2}$; les cornes de fer portées par le faux prophète Sédécias ${ }^{3}$; Ézéchiel ligoté et muet ; ses cheveux et sa barbe rasés ${ }^{4}$, etc. Ou bien qu'ils utilisaient comme des métaphores le détournement des pratiques sociales en usage de leur temps : la nudité provocatrice; le mariage avec une prostituée ${ }^{5}$, etc.

Dans une série d'articles essentiels sur la prophétie biblique et sa mise par écrit, Cristiano Grottanelli a montré comment la prophétie dans le judaïsme ancien se singularisait par rapport à la norme en usage au sein du Proche-Orient, en privilégiant l'expression orale et en s'opposant à la formulation écrite et au scribe ${ }^{6}$. Les livres prophétiques les plus anciens dans la Bible, comme Amos ou Osée, attestent d'un univers dont toute idéologie ou terminologie de l'écriture sont absentes : « un monde de pure oralité », dit Grottanelli'. À l'inverse les très anciennes archives prophétiques de Mari (XVIII ${ }^{\mathrm{e}}$ siècle avant l'ère vulgaire) ou les tablettes de prophéties assyriennes du $\mathrm{VII}^{\mathrm{e}}$ siècle témoignent que, dans ces deux cultures, les prophéties sont systématiquement mises par écrit avant d'être transmises au souverain. On ne sera donc pas surpris de trouver trace, dans la Judée des $\mathrm{VIII}^{\mathrm{e}}$ et $\mathrm{VII}^{\mathrm{e}}$ siècles, d'une confrontation

1. $1 \mathrm{R} 13$.

2. 1 R 20, 35-38.

3. 2 Ch 18,10

4. Éz 3, 25-26; 5, 1-4.

5. Os 1,2 .

6. Grottanell 1999, 2001 et 2003.

7. «In the most ancient Hebrew prophetic books, such as Amos and Hosea, the written texts contains neither an ideology nor a terminology of writing. The world is one of pure orality. » (GROTTANELLI 1999, p. 176). 
entre d'une part ces grands maîtres de l'oralité que sont les prophètes hébreux, de l'autre la nouvelle technique de savoir et de pouvoir que constitue l'écriture. Cette époque est en effet celle de l'apparition et de l'essor d'une « classe » de scribes en Judée ou, si le mot paraît trop fort, d'un groupe socialement identifié et assez important de spécialistes de l'écriture ${ }^{8}$. Tout indique que la technique nouvelle de l'écriture a d'abord servi aux prophètes de l'ancien Israël, de support à leurs démonstrations métaphoriques, rituelles et symboliques.

On trouve les premières traces de cet usage différencié de l'écriture chez le prophète, ou le groupe de prophètes, connu sous le nom de «premier» ou « proto-Isaïe ». Ce nom désigne l'auteur, individuel ou collectif, des 39 premiers chapitres du livre biblique d'Isaïe.

Prophète dans la Jérusalem de la fin du VIII ${ }^{\mathrm{e}}$ siècle avant l'ère vulgaire (c. 740-700), il assiste aux prémices politiques et guerrières des événements qui conduiront à la disparition du royaume de Juda.

Les passages du proto-Isaïe traitant de l'écriture des prophéties sont assez peu nombreux et bien identifiés : Is $8,1-4$ et $16-20 ; 29,11-12 ; 30,8-14$ et 34,16 . On peut classer ces textes en deux séries : ceux qui supposent déjà acquise l'existence d'un recueil écrit, même embryonnaire, des prophéties d'Isaïe ; ceux qui évoquent le processus d'écriture d'une prophétie et se

8. L'existence des scribes et des écoles de scribes dans l'Israël ancien monarchique a donné lieu à un vigoureux débat international dans les années 1970-1980. Deux points semblent établis : 1/ Toutes les données, épigraphiques, archéologiques et historiques convergent pour attester d'une importante activité scribale en Judée à la fin de l'époque monarchique, c'est-à-dire au VIII ${ }^{\mathrm{e}} \mathrm{s}$. av. : ostraca témoignant d'un apprentissage organisé, donc d'écoles de scribes; abondance des bulles-sceaux, attachées au papyrus et portant les noms des auteurs et/ou des témoins d'actes juridiques ; émergence d'une première littérature de sagesse (les Proverbes) reflétant la tradition ou l'idéologie des scribes. 2 / Cet essor des scribes et le développement du recours à l'écrit sont des phénomènes nouveaux en Judée. Partant de l'hypothèse pertinente que l'existence d'une classe de scribes et/ou de bureaucrates dépendait du niveau de développement économique du pays ainsi que du degré de centralisation de l'État - en d'autres termes du besoin qu'on en a, et de la capacité à les entretenir David Jamieson-Drake s'est efforcé de collecter toutes les données archéologiques disponibles dans ce domaine. Travail difficile et délicat pour toutes sortes de raisons évidentes mais qui, bien que Jamieson-Drake demeure très prudent dans ses conclusions, aboutit à ceci : c'est seulement au cours du VIII $\mathrm{e}$. que la Judée devient un État monarchique développé ayant les moyens de recourir à une classe spécialisée d'administrateurs-scribes. Voir JAMIESON-DRAKE 1991. situent donc en amont de l'existence d'un tel recueil. Si l'on retient le mot sêfer (« livre ») comme critère discriminant, sa présence dans le texte attestant l'existence du recueil en question, on constate que les chapitres 29, 30 et 34 relèvent de la première série, tandis que le chapitre 8 appartient à la seconde. C'est aussi celui qui propose les passages les plus intrigants. Commençons par Is 8, 1-4 : «Iahvé me dit: "Prends une grande tablette et écris dessus avec un poinçon ordinaire: Mahēr-Šălāl-Haš-Baz. Puis, fais témoigner à mon sujet des témoins sûrs, Ouriyah, le prêtre et Zekaryahou, fils de Yebérékyahou." Je m'approchai ensuite de la prophétesse, elle conçut et enfanta un fils. Iahvé me dit : "Nomme-le Mahēr-Šālāl-Haš-Baz, car avant que le garçon ne sache appeler « mon père » et « ma mère », on transportera les richesses de Damas et le butin de Samarie devant le roi d'Assur." " "

L'épisode se situe à l'époque de la guerre «syroephraïmite », vers 735-733. Isaïe s'oppose à la politique du roi de Juda, Achaz quand celui-ci sollicite le soutien de l'Assyrie contre la coalition des rois de Damas et d'Israël, dirigée contre la Judée. La position d'Isaïe est que la coalition des deux royaumes du nord sera nécessairement vaincue par les Assyriens, et qu'il est donc à la fois inutile et maladroit d'appeler ceux-ci au secours et de s'en rendre ainsi délibérément tributaire. Comme sa prophétie n'a pas été écoutée par le roi Achaz, Isaïe a recours à la démonstration publique qui nous est rapportée ici : il écrit devant témoins le nom de son fils à naître, ce nom étrange mais signifiant, Mahēr-Šălāl$H a s ̌-B a z$, dont la traduction est à peu près « Vite au butin ! Tôt au pillage ! », un cri de guerre hébreu signifiant la déroute complète des ennemis. Le sens en est aussitôt explicité ; avant que cet enfant ne sache parler les deux rois du nord auront été vaincus.

Isaïe recourt donc ici à deux méthodes d'exposition métaphorique, pour illustrer et compléter la prophétie orale : la première, que nous avons déjà mentionnée, consiste à détourner un usage social à des fin prophétiques ; ici la conception et la naissance d'un fils ainsi que le nom que son père lui donne ${ }^{10}$. Le second procédé est en revanche absolument nouveau : c'est le recours à l'écriture. Pourquoi l'écriture ? Pour conserver et témoigner? Mais il est fait appel à des témoins fiables et incontestables. Pour dissimuler? Mais le geste est fait publiquement, à la vue de tous, sur un " grand » support. Il faut donc regarder de plus près les gestes d'Isaïe quand il s'empare de la technique des scribes.

9. Traduction DHORME 1959.

10. Isaïe a déjà eu recours à ce même procédé lorsqu'il a nommé son premier fils Emmanuel, voir Is 7, 14. 


\section{Sur quoi écrit-il et avec quel instrument?}

Is $8,1:$ «YHWH m'a dit : prends-toi un grand gillāyôn et écris dessus avec un heret ordinaire ${ }^{11}$. »

Premier point : le verbe Kātab employé ici renvoie spécifiquement à l'écrit, pas à une activité voisine comme « graver » ou « dessiner». Au Proche-Orient à l'époque d'Isaïe, trois façons d'écrire prédominent : a/ l'inscription au moyen d'un poinçon sur un support tendre, argile ou cire, dont les cunéiformes constituent l'archétype, est longtemps demeurée le système dominant. b/. En Judée il est déjà remplacé par la cursive, utilisant de l'encre et un instrument tendre (calame, roseau, bâton) sur divers supports, comme les papyrus, ostraca ou peaux. c/ Enfin, les textes officiels et/ou importants sont gravés, en utilisant un burin sur un support résistant et durable, la pierre ou le métal. L'écriture adoptée par Isaïe relève manifestement du troisième registre.

En effet le prophète écrit ici au moyen d'un heret. Qu'est-ce donc qu'un heret ? Le mot ne figure que deux fois dans la Bible hébraïque : ici et dans le récit de l'Exode où Aaron construit le veau d'or avec tout l'or recueilli auprès des Hébreux au désert.

Ex 32, 4a : « Il le prit [l'or] de leurs mains, puis le façonna avec un heret et en fabriqua un veau de métal fondu ${ }^{12 . »}$

Il s'agit donc d'un outil permettant de travailler le métal. Dans le cas d'Isaïe, les traducteurs français recourent ordinairement au mot «stylet»; mais un autre mot existe en hébreu pour désigner l'instrument dont se servent les scribes pour écrire, ce à quoi renvoie précisément le mot «stylet» : cela se dit 'êt et on en trouve plusieurs attestations dans la Bible ${ }^{13}$.

Deux passages tirés des manuscrits de Qumrân (dans l'un des Hymnes du Maître de Justice et dans le Règlement de la guerre) donnent à penser que le heret désigne un outil spécialement utilisé pour graver des textes : il y est question des décrets divins gravés pour l'éternité. On trouve par exemple dans le Règlement de la guerre (1QM XII 3) : « Tu les as gravés à leur intention, avec le heret de vie pour régner [...] à toutes les époques des temps éternels. ».

Dans ce passage, c'est YHWH qui est censé avoir gravé ses volontés pour l'éternité. Dans l'hymne c'est l'univers tout entier et la division du temps en périodes

11. Wayy'ōmer YHWH 'ēlay qah-l'kā gillāyôn gādôl $\hat{u} k^{\circledR} t o ̄ b$ 'ālā $(y) w$ b hereț' 'enôs.

12. Wayyiqqah miyyādām wayyāṣar 'ōtô baḥheret wayya'áséhû 'ēgel massēkāh.

13. Voir, inter al., Jr 8, 8 et 17, 1 ; Job 19, 24 ; Ps 45, 2. assignées qui sont gravés pour l'éternité devant $\mathrm{YHWH}^{14}$. Dans la littérature communautaire de Qumrân cette image des tablettes célestes où sont " gravés » les décrets divins exprime avec force le caractère immuable de ces décrets, dans l'esprit du rigoureux prédéterminisme qumrânien. Tout concourt donc à faire du heret, qu'on le traduise par « stylet» ou « burin », un instrument destiné à graver durablement des textes importants, sur un support de pierre ou de métal.

Car c'est la deuxième question : qu'est-ce que ce grand gillāyôn sur lequel Isaïe grave le nom prophétique de son fils à naître ? La plupart des traducteurs, reprenant ici les versions (grecques, latines et araméennes) ${ }^{15}$, le comprennent comme un rouleau ou une tablette, les supports traditionnels de l'écriture à cette époque ${ }^{16}$. Cela n'est guère satisfaisant dans la mesure où le stylet du prophète semble d'abord destiné à graver sur du métal.

Le mot gillāyôn n'apparaît que deux fois dans la Bible hébraïque, les deux fois dans Isaïe : ici et dans un passage où le prophète condamne le luxe des femmes de Jérusalem. «Ce jour-là, dit la prophétie, Adonaï arrachera... » (Is 3, 23). Suit une description minutieuse des atours, bijoux, vêtements et colifichets féminins à la mode en ces années-là, qui seront ôtés l'un après l'autre aux Hiérosolymitaines élégantes de sorte qu'elles se retrouveront finalement nues, chauves et humiliées - expression du sadisme ordinaire du fanatisme viril. Dans cette liste des ornements condamnés figure un verset où il est question de gilyōnîm, pluriel de gillāyôn.

Is 3, 23: «Ainsi que les gilyōnîm, les pièces de lin, les turbans et les châles ${ }^{17}$. »

Le contexte et les arguments philologiques ont conduit les traducteurs modernes, depuis Gesenius, à comprendre qu'il s'agissait ici de miroirs, c'est-à-dire alors de plaques de métal poli ${ }^{18}$. Dès lors le seul mystère réside dans la constance des traducteurs et commentateurs d'Isaïe à traduire d'abord gillāyôn par « miroir »

14. Hymnes IX 24 (1QH $\left.\mathrm{QH}^{\mathrm{a}} 24\right)$.

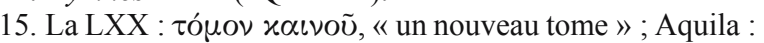
$\delta \iota \varphi \theta \varepsilon ́ \rho \omega \mu \alpha$ (difterôma) « une peau apprêtée »; Symma-

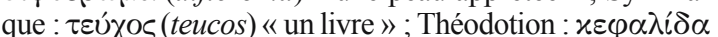
(kefalida), " un chapitre »; le Targum : lûah rab, " une grande tablette »; la Vulgate : librum, « un livre».

16. Seule la Traduction Ecuménique de la Bible (TOB) y a vu un "sceau-cylindre», sur la base d'une étymologie $(\mathrm{gll})$ à vrai dire peu convaincante.

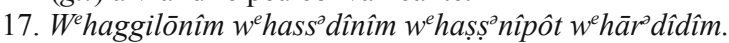

18. L'alternative, fondée sur la LXX et l'existence d'une racine voisine en arabe classique, qui conduirait à y voir des voiles de lin, serait peu compatible avec le passage que nous étudions, dans lequel Isaïe " grave » le nom de son fils. 
quand il s'agit des élégantes de Jérusalem, puis par « tablette»-dont ils précisent volontiers si elle était de pierre ou de bois (?), dès lors qu'il est question du support sur lequel Isaïe a écrit. Il me paraît plus cohérent d'admettre qu'Isaïe a gravé le nom de son fils sur un grand miroir de métal poli et de lire ainsi Is 8,1 : «YHWH m'a dit : procure-toi un grand miroir et écris dessus avec un simple burin. »

Il me paraît donc établi qu'Isaïe ne s'attachait pas, en l'occurrence, aux fonctions triviales et traditionnelles de l'écriture : témoigner, archiver, transmettre. J'inclus dans cette série les interprétations de type politique, qui ont la faveur de la plupart des commentateurs contemporains, et selon lesquelles le prophète aurait eu recours à l'écrit dans une circonstance critique, alors que son avis n'avait pas été pris en compte par le pouvoir ${ }^{19}$.

L'apparat dont Isaïe entoure le geste de graver sa prophétie, les ustensiles précieux (un miroir) et les témoins prestigieux auxquels il a recours, donnent à toute l'opération un caractère cérémoniel ; une cérémonie dans laquelle l'écriture elle-même devient un élément du rite - si l'on peut nommer « rite», sans choquer, un acte prophétique. Pourtant on se heurte ici à une difficulté d'ordre anthropologique : car si l'on perçoit bien que l'action de graver le nom d'un enfant à naître sur un miroir n'a rien d'anodin, on ne voit pas (du moins pas encore) quelle portée symbolique cela pouvait avoir dans le contexte historique et culturel de la Judée du VIII ${ }^{\mathrm{e}}$ siècle.

On connaît en revanche l'une des conséquences que le prophète attendait de son geste. Dans le même chapitre 8 et, dans la rédaction initiale, immédiatement après l'épisode du miroir gravé, figure cette instruction d'Isaïe.

Is $8,16:$ : Renferme cette attestation; scelle cette instruction en présence de mes élèves ${ }^{20}$. »

Ce qui est décrit ici, c'est le scellement d'un acte juridique effectué en présence de plusieurs témoins. Les mots importants ici sont tôrāh et $t^{\natural} \hat{u} d a h$. Torah, chez le proto-Isaïe ne doit pas s'entendre comme « la Loi »; il conserve son sens premier «d'instruction », en l'occurrence d'instruction prophétique, c'est-à-dire la parole de YHWH transmise à son peuple par l'intermédiaire du prophète ${ }^{21}$. La $t^{2} \hat{u} d a h$ est un mot rare dans

19. Voir par exemple le commentaire de BLENKINSOPP 2000, p. 414-416 sur ce passage et sur Is 30,8 .

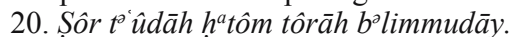

21. "Tôra a term for prophetic teaching in the first major section of the book of Isaiah $(1: 10 ; 2: 3 ; 5: 24 ; 30: 9) »$, BLENKINSOPP 2000, p. 243. la Bible hébraïque : il n'y apparaît que trois fois, deux fois dans ce chapitre d'Isaïe, et une troisième fois en Rt 4, 7. La racine du mot, comme l'usage qui en est fait dans Ruth, établit qu'il s'agit d'un document à portée juridique, validé en présence de témoins ${ }^{22}$. On est ici dans le cadre de l'idéologie de l'Alliance, et non dans une tentative de communication: l'écrit, scellé et fermé, n'est donc pas spontanément conçu comme un moyen de transmettre la prophétie. Ce qui importe au prophète c'est la transformation, au moyen d'une série de gestes connus et pratiqués par la société de son temps, de sa tôrāh en une $t^{\circ} \hat{u} d a h$, qui engage la divinité et son peuple dans un rapport contractuel. L'écriture est l'un de ces gestes convenus, la présence des élèves et le scellement du rouleau en sont deux autres. On est revenu à l'usage métaphorique des pratiques sociales, en l'occurrence le contrat ${ }^{23}$.

Pour le proto-Isaïe, l'écriture apparaît donc d'abord comme une fonction sociale dont les prophètes peuvent faire tout usage métaphorique et/ou symbolique qui leur permettra de renforcer la puissance convaincante de la prophétie. Au contraire des scribes, des prêtres et des dirigeants administratifs et politiques de l'État, Isaïe ne lui reconnaît manifestement aucun caractère d'innovation épistémologique et moins encore de statut privilégié dans la relation avec l'au-delà.

Le conflit autour de l'écriture entre prophètes et scribes s'est poursuivi probablement jusqu'à la chute de Jérusalem et du royaume de Juda devant les armées babyloniennes. En attestent plusieurs passages du prophète Jérémie. Grottanelli s'est ainsi surtout intéressé au prophète Jérémie pour la raison qu'il lui semblait pouvoir y repérer le moment où la prophétie hébraïque, jusqu'alors essentiellement orale, avait dû apprendre à composer, non sans conflits, avec l'univers de l'écriture et avec ses professionnels et spécialistes, les scribes ${ }^{24}$.

22. Traduction Ecuménique de la Bible, p. 766-767: «(...) désigne en Rt 4, 7 le geste symbolique par lequel on validait, à défaut d'écrit, un accord passé devant témoins. » WATTS 1985 , p. 122 : « The basic meaning of the root is "to give testimony", "to be a witness". » BLENKINSOPP 2000, p. $247:$ « $T^{\mathrm{c}} \hat{u} d a h$ signifies a text validated by witnesses (cf. Ruth 4:7). »

23. Peut-être faut-il aussi chercher un élément d'explication du côté de cet étrange verset du même chapitre où Isaïe se qualifie, lui-même et ses enfants, de «signes» en Israël. Is 8,18a : «Voici que moi-même et les enfants que YHWH m'a donnés sommes comme des signes et comme des signaux en Israël » hinnēh 'ānōkî w'ha(y)y $y^{\curvearrowright}$ ādîm 'ă šer nātan-lî YHWH l'ōôtôt ûl'môptîm b’yiśrāēel.

24. On se reportera à GROTTANELLi 1999, 2001 et 2003, en particulier au dernier dans lequel l'auteur analyse ce passage extraordinaire de Jérémie où le prophète condamne la " tôrāh mensongère » des scribes (Jér 8, 8-9). Inutile de souligner combien cette idée formulée par un prophète 


\section{LE ROULEAU BRÛLÉ DE JÉRÉMIE}

Durant un hiver dans Jérusalem, le roi Yoyakim, fils et deuxième successeur du grand réformateur deutéronomiste Josias, placé sur le trône de la Judée par le pharaon d'Égypte, fait interdiction au prophète Jérémie de poursuivre ses vaticinations en public dans la cour du Temple de Jérusalem, comme il en avait pris l'habitude. Le roi apprend bientôt que Jérémie a alors dicté ses prophéties au scribe Baruch, lequel les a lus à son tour à haute voix dans l'enceinte du Temple. Le roi se fait apporter cet objet nouveau, un rouleau écrit reproduisant des prophéties, afin qu'on le lise devant lui et devant ses proches conseillers. Au cours de cette lecture le roi se livre à une étrange activité décrite en ces termes (Jr 36, 21-25) : «Le roi envoya Yehudi chercher le livre. Yehudi le prit dans la chambre d'Élishama le scribe et le lut aux oreilles du roi et à celles de tous les notables autour du roi. Le roi siégeait dans son palais d'hiver, au dix-neuvième mois [c'est-à-dire, kislev, approximativement décembre-janvier], et un brasier flambait devant lui. Quand Yehudi avait lu 3 ou 4 "colonnes", le roi découpait le livre avec une lame et le jetait dans la flamme du brasier, jusqu'à ce tout le rouleau fut brûlé dans le feu. Le roi et tous ses serviteurs qui entendirent toutes ces paroles ne furent pas effrayés et ne déchirèrent pas leurs vêtements. Bien qu'Elnathan, Delayahû et Gemaryahû fussent intervenus auprès du roi pour qu'il ne brûlât pas le rouleau, il ne les écouta pas. »

On doit bien comprendre qu'il se passe ici quelque chose de particulier et d'important: le roi lui-même, contre l'avis de ses proches conseillers, se charge de brûler le rouleau des prophéties au fur et à mesure que son scribe les lui lit.

\section{Qu'est-ce qui se passe là exactement ?}

On peut d'abord écarter un certain nombre de fausses pistes : il ne s'agit pas d'un geste anodin, de colère ou de folie de la part du roi. En effet, il y a un débat politique sur le brûlement: c'est donc une décision réfléchie. Ensuite le geste est répété systématiquement et sans hâte jusqu'à l'accomplissement. L'indication des «trois à quatre colonnes $\gg{ }^{25}$ lues entre deux découpages du

canonique, que la Tôrāh non moins canonique pût être « mensongère », a semé un certain trouble chez la plupart des commentateurs - qui s'en débrouillent en général en glissant discrètement le problème sous le tapis.

25. Delet, plur. d’lātôt : porte, battant de porte, d'un bâtiment, d'une ville ou du ciel - d'où, dans ce cas l'usage du duel. Métaphoriquement : couvercle, mâchoires d'un crocodile, lèvres ; couvercle. Et ici : colonnes du manuscrit; dans ce sens de « colonnes » c'est un hapax biblique. rouleau correspond à la largeur ordinaire d'un rouleau déployé pour la lecture ; ce détail, comme celui de l'usage d'une lame, contribuent à représenter un roi maître de ses choix et de ses gestes. D'ailleurs le brûlement n'interrompt pas la communication : le roi est ses conseillers écoutent jusqu'au bout les paroles prophétiques.

En dépit de ce caractère répétitif du geste, je ne crois pas non plus qu'on soit dans le registre du rite ; toute l'affaire manque trop de sacré pour cela : bien que le brûlement du rouleau sur un brasier puisse évoquer l'image des "consumations" sacrificielles, tout le récit nous détourne de cette comparaison en insistant sur le caractère profane de la situation : l'histoire se déroule au palais du roi et non au Temple; le feu est à usage domestique (il fait froid); on observe enfin l'absence de prêtres parmi les conseillers présents.

Rien n'indique dans ce récit que le roi, ses conseillers, ou d'ailleurs Jérémie lui-même, aient su lire; l'écriture et la lecture y apparaissent au contraire comme des opérations confiées à une espèce de techniciens spécialisés, nommés les scribes : Baruch, chargé par Jérémie d'écrire ses paroles ; Yehudi, chargé par le roi de lui lire le rouleau. Comme les premières automobiles n'allaient pas sans leur chauffeur-mécanicien, les premiers écrits prophétiques d'Israël ne vont pas sans leur scripteurlecteur humain. Si on voulait empêcher quelqu'un de faire un trajet en voiture, il était plus efficace de brûler son automobile que de tuer l'un de ses chauffeurs... à moins, bien sûr qu'il n'ait possédé plusieurs voitures, comme le roi Yoyakim va s'en apercevoir lorsque Jérémie aura dicté à nouveau ses prophéties à Baruch sur un second rouleau. Il ne faut jamais pousser trop loin les comparaisons: dans notre histoire ce n'est évidemment pas le rouleau qui importe mais les paroles prophétiques.

La question : pourquoi le roi Yoyakim a-t-il brûlé le premier rouleau? Il n'est jamais présenté comme un simple d'esprit qui aurait pu faire la confusion entre le contenu (les paroles prophétiques) et le support (le rouleau).

L'hypothèse très féconde de Grottanelli est que, dans ce moment critique du passage de la prophétie israélite de l'oral à l'écrit, l'objet qui en résultait (ici le rouleau écrit par Baruch) se situait à la fois en deçà et au-delà de l'écriture ${ }^{26}$.

En deçà : parce qu'il conserve un caractère d'oralité fondamentale : il n'est écrit, sous la dictée, que pour atteindre les oreilles du peuple à qui Jérémie ne peut pas s'adresser lui-même. De ce caractère «oral» atteste encore la capacité de Jérémie à le dicter à nouveau en entier, identique au premier texte. Mais aussi au-delà : parce que l'objet rouleau-écrit semble avoir acquis en

26. Grottanelli 1999. 
lui-même en tant qu'objet une puissance propre, que Grottanelli nomme une « valeur magico-symbolique », évidemment liée à la parole de YHWH.

Ce serait donc cette efficacité "magico-symbolique » du rouleau que le roi Yoyakim s'efforcerait de détruire en le brûlant.

Il est certain que le passage des prophètes à l'écrit s'opère à grand renfort de symbolisations du geste prophétique et du medium prophétique - en particulier la personne même du prophète et de sa famille, comme dans le cas évoqué plus haut du proto-Isaïe. La démonstration de Grottanelli prend toute sa force quand il s'appuie par exemple sur l'épisode de Jér 51, 59-64 : Jérémie envoie aux exilés de Babylone un rouleau sur lequel sont écrites des prophéties contre la ville païenne ; il recommande que ces prophéties soient lues au bord du fleuve puis que le rouleau en soit noyé dans l'Euphrate. " The passage in question is an exemplary case of materialization of the message in order to mediate between prophetic word and efficacious symbolic action ${ }^{27}$. " Le caractère " magico-symbolique » $\mathrm{du}$ rouleau sur lequel sont inscrites les prophéties apparaît ici indiscutable.

En revanche il n'en va pas de même dans l'épisode du rouleau brûlé à Jérusalem. Au contraire le récit s'efforce de débarrasser le geste du roi de tout caractère rituel ou sacré. On se situe plutôt dans l'ordre du politique, souligné par l'opposition topographique et symbolique entre le Temple et le Palais.

Rappelons le parcours de ce rouleau : Jérémie a dicté ses prophéties à Baruch ; Baruch a lu ces prophéties en public dans l'enceinte du Temple, depuis la chambre du scribe (fils du scribe du Deutéronome); son fils, le petit-fils du scribe du Deutéronome s'est rendu au Palais répéter ces paroles, de mémoire, aux conseillers du roi ; Baruch, convoqué par les conseillers, a lu devant eux le rouleau; les conseillers se sont inquiété de savoir comment le rouleau a été écrit puis l'ont confisqué et ont renvoyé Baruch ; ils sont ensuite allé répéter les prophéties, de mémoire, au roi ; enfin, le roi envoie chercher le rouleau et se le fait lire. On voit que la prophétie a effectué un va-et-vient continuel entre l'oral et l'écrit, entre lecture et récitation. L'affaire n'apparaît pas centré sur cette question du support.

\section{De quoi s'agit-il alors ?}

À mon avis on est ici dans une querelle des tôrāh. On sait que, chez Isaïe et Jérémie, le mot tôrāh a souvent conservé sa signification ancienne de « enseignement, instruction », plus particulièrement tirés des prophéties ${ }^{28}$. Un passage de Jérémie, où il évoque ses ennemis judéens est ici particulièrement significatif : « Ils dirent alors : "Allons, nous inventerons des inventions contre Jérémie ; car la tôrāh ne disparaîtra pas chez les prêtres, ni la délibération chez les sages, ni la parole chez les prophètes"29. " À mon sens, le prophète énonce ici les trois catégories connues de production de la connaissance, dans la Judée de la fin de l'époque monarchique : la tôrāh des prêtres - mise par écrit; la réflexion

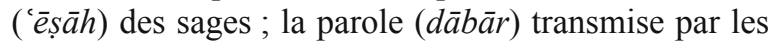
prophètes. Il faut entendre cette énonciation comme une suite croissante dans le domaine de la connaissance, autrement dit comme une théorie de la connaissance des volontés divines, dans laquelle la sagesse l'emporte sur la tôrāh, et la parole (divine) sur les deux autres.

Le roi Yoyakim est le fils de Josias, « l'inventeur de la Torah » (le Deutéronome). Il défend donc très matériellement, en brûlant le rouleau de Jérémie, l'idée qu'il ne peut exister deux écritures normatives concurrentes, deux paroles écrites de YHWH contradictoires. La querelle des normes, ou la querelle des autorités qui pouvait exister parole contre parole (le prophète contre le roi) ne doit pas se poursuivre écrit contre écrit (la tôrāh du prophète contre la tôrāh légale) : c'est cela qu'opère Yoyakim dans la sphère de compétence qui est la sienne. En brûlant le rouleau il sait parfaitement qu'il ne détruit pas la prophétie ; mais il veut détruire l'autorité que la prophétie prétend s'approprier en usurpant la forme écrite qui avait fondé l'autorité de la Loi. C'est donc bien d'un rite qu'il s'agit en fin de compte. Mais d'un rite qui ne renvoie pas au sacré, d'un rite politique qui s'improvise pour exprimer la souveraineté de la Loi du roi.

Université de Lille III
28. Voir Is 1,$10 ; 2,3 ; 5,24 ; 8,16.20 ; 24,5 ; 30,9$. Et Jér 2 , $8 ; 6,19 ; 8,8 ; 9,12 ; 16,11 ; 18,18 ; 26,4 ; 31,33 ; 32$, $23 ; 44,10$. Voir en particulier la critique de la «torah mensongère » des scribes (Jér 8, 8-9) déjà mentionnée ci-dessus, ainsi que GROTTANELLI 2003.

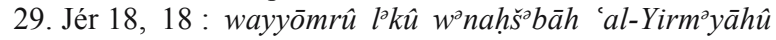

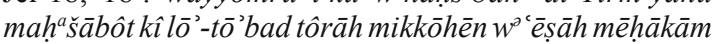
$w^{\curvearrowright} d \bar{a} b \bar{a} r$ minnābî̀. 


\section{BIBLIOGRAPHIE}

BLENKINSOPP J.

2000 Isaiah 1-39. A New Translation with Introduction and Commentary, New York.

DHorme É. (trad.)

1959 La Bible. Ancien Testament, vol. 2, Paris.

GrotTANELLI C.

1999 « Prophecy and Writing in the Ancient Near East», dans C. Grottanelli (éd.), Kings and Prophets. Monarchic Power, Inspired Leadership, and Sacred Text in Biblical Narrative, New York, p. 173-183 (traduit de l'italien, 1982).
2001 «La scrittura nell'ambiente della Bibbia. Valori culturali e religiosi dello "scritto" nel contesto storico che a generato l'Antico Testamento », Riccerche Storica Bibliche 13/1, p. 11-26.

2003 «On Written Lies", dans M. FINKELBERG et G. Stroumsa (éd.), Homer, the Bible and Beyond. Literary and Religious Canons in the Ancient World, Leyde, p. 53-62.

JAMIESON-DRAKE D. W.

1991 Scribes and Schools in Monarchic Judah. A SocioArcheological Approach, Sheffiled.

WATTS J.D.W.

1985 Isaiah 1-33, Waco: Word Books (Word Biblical Commentary 24). 